\title{
Investigating the affinity of BDE154 and 3OH-BDE154 with HSA: Experimental and simulation validation
}

\author{
Wu Yang ${ }^{\mathrm{a}}$, Lulu Yang ${ }^{\mathrm{a}}$, Zhongsheng $\mathrm{Yi}^{\mathrm{a}}{ }^{\mathrm{*}}$, , Zhiwei Wu $^{\mathrm{a}}$, Jinfang Nie ${ }^{\mathrm{a}}$, Aiqian Zhang ${ }^{\mathrm{b}}$ \\ a Guangxi Colleges and Universities Key Laboratory of Food Safety and Detection, Collaborative Innovation Center for Water Pollution Control and Water \\ Safety in Karst Area, College of Chemistry and Bioengineering, Guilin University of Technology, Guilin 541004, China \\ b State Key Laboratory of Environmental Chemistry and Ecotoxicology, Research Center for Eco-Environmental Sciences, Chinese Academy of Sciences, \\ Beijing 100085, China
}

\section{A R T I C L E I N F O}

\section{Article history:}

Received 20 September 2016

Received in revised form 11 February 2017

Accepted 2 March 2017

Available online 6 March 2017

\section{Keywords:}

Human serum albumin

Binding studies

Circular dichroism

Molecular dynamics

\begin{abstract}
A B S T R A C T
The physicochemical properties of polybrominated diphenyl ethers are important for modeling their transport, but these data are often missing. Here, satisfactory bioactivity results were obtained using human serum albumin as the carrier, 2,2',4,4',5,6'-hexabromodiphenyl ether (BDE154) and 3-hydroxy$2,2^{\prime}, 4,4^{\prime}, 5,6^{\prime}$-hexabromodiphenyl ether (3OH-BDE154) as the ligands, using UV-visible absorbance, fluorescence, circular dichroism, molecular docking, and molecular dynamics methods. The interactions between human serum albumin and BDE154 or 30H-BDE154 were verified, consistent with the static quenching procedure. At pH 7.4, the binding constants of the complexes for site I were relatively comparable and increased in the order BDE154 $<30 \mathrm{H}-\mathrm{BDE} 154$. Then, the secondary structure and kinetic parameters of albumin were analyzed using the circular dichroism spectra and GROMACS software. The data obtained from these simulations indicate that hydrophobic attraction might be the key factor for the stability of complexes. The docking experiments provided further insight into the hydrophobic pocket and showed that 30H-BDE154 has a stronger binding affinity to human serum albumin than BDE154. The experimental spectral data were obtained and compared with the simulation results, showing good agreement. A detailed analysis of PBDEs-HSA interactions would provide valuable information to better understand the interaction on this class of compounds.
\end{abstract}

(c) 2017 Elsevier B.V. All rights reserved.

\section{Introduction}

Human serum albumin (HSA) has been widely used as a model protein for studying the interactions between proteins and different substrates (Mao et al., 2001; Wang and Ni, 2014; Sato et al., 2014). The advantage of this protein-based receptor is that HSA is a relatively simple protein for determining its binding mode and identifying the possible binding site(s). In the past few years, HSA-ligand interactions have been found in drugs (Shahlaei et al., 2015), environmental pollutants (Wang and Zhang, 2015), and dyes (Wu et al., 2015), etc. However, the majority of these studies are performed from a global perspective. The site-specific capture of HSA-ligand interactions under physicochemical conditions has been reported. In such cases, different types of weak interactions have been observed, e.g., hydrophobic effects, H-bonding, $\pi$-stacking, and electrostatic interaction (Carter and He, 1990; Baler

\footnotetext{
* Corresponding author. Fax: +86 7735896839.

E-mail address: yzs@glut.edu.cn (Z. Yi).
}

et al., 2014). It is owing to that HSA contains a single intrinsic tryptophan residue at position 214 in subdomain IIA (namely Sudlow's site I), whose fluorescence is sensitive to the ligand bonded nearby (Ghuman et al., 2005; Yadav et al., 2014; Wu et al., 2016). Moreover, most of the compounds located within the cavities in the two sites have an affinity in the range $10^{4}-10^{6} \mathrm{M}^{-1}$ (Zaidi et al., 2013; Zunszain et al., 2008).

Over the past decades, polybrominated diphenyl ethers (PBDEs) have been widely used as additives inflame-retardant compounds in diverse consumer products because of their high lipid solubility and chemical stability (McDonald, 2002; La Guardia et al., 2006; Stapleton et al., 2011). However, PBDEs are not chemically bound to the products and therefore enter the environment (Odusanya et al., 2009). A long-term exposure to PBDEs is a possible health risk for humans because they have been shown to induce neurotoxic and endocrine-disrupting effects in experimental animals. Moreover, in vivo and in vitro laboratory studies have confirmed that PBDEs produce hydroxylated BDE congeners (OH-BDE) in hepatocytes for secondary pollution (Chen et al., 2013; Erratico et al., 2013). Moreover, two recent studies on PBDEs and thyroid hormones also 
<smiles>Brc1cc(Br)c(Oc2cc(Br)c(Br)cc2Br)c(Br)c1</smiles>

BDE154<smiles>Oc1c(Br)c(Br)cc(Oc2c(Br)cc(Br)cc2Br)c1Br</smiles>

3OH-BDE154

Fig. 1. Structures of BDE154 and 3OH-BDE154.

involved hydroxylated PBDEs (OH-PBDEs), because OH-PBDEs are structurally more similar to PBDEs and have a stronger binding capability to human transthyretin (Cao et al., 2010; Dingemans et al., 2011; Marchesini et al., 2008; Zota et al., 2011). Unfortunately, the data for the properties of OH-PBDE congeners (Wan et al., 2010) are very limited, and relatively few interaction metabolites have been identified between OH-PBDEs and HSA.

BDE154 is a hexabrominated congeners, it is one of the most abundant PBDEs that has been detected in tissues, human blood, breast milk, etc. And BDE154 has been demonstrated to be severely toxic to human beings and animals (Wang et al., 2016). In the past few years, there is a lack of information studied on BDE154, but its residual level in the environment and human body is going up. According to the present researches (Lílian et al., 2014; Alecsandra et al., 2016), BDE-154 impairs mitochondrial functioning and induces apoptotic death of human hepatoblastoma cell line. Also, as the metabolites of the BDE154, 30H-BDE154 is still toxic in the human body and environment because of the chemical structure of which is similar to the parents.

In this study, the thermodynamics, aggregation characteristics, and surface properties of two binding models for BDE154 and 3OHBDE154 were investigated (Fig. 1). The objectives of this study were as follows:(i) to combine an unrestrained all-atom molecular dynamics (MD) simulation and other computational methods to provide insights into how the metabolites of PBDEs/OH-PBDEs bind to HSA and activate it from the conformational transition perspective, (ii) to elucidate the effects of hydrophobic and electrostatic interactions using the globular protein HSA, and (iii) to obtain the binding parameters using UV-visible absorbance, fluorescence, circular dichroism (CD), activity assay, and molecular docking methods. Excellent agreement was observed between the computer simulation results and experimental spectral results, thus guaranteeing the accuracy of the study.

\section{Materials and methods}

\subsection{Materials}

HSA ( $99 \%$, pure free fatty acid, A1887) was purchased from Sigma-Aldrich, USA. A stock solution of $10^{-5} \mathrm{M}$ in Tris- $\mathrm{HCl}$ buffer ( $\mathrm{pH} 7.4$ ) was prepared and stored in the dark. The ligands, $2,2^{\prime}, 4,4^{\prime}, 5,6^{\prime}$-hexabromodiphenyl ether (BDE154) (50 $\mathrm{g} \mathrm{mL}^{-1}$ in isooctane, bde154s) and 3-hydroxy-2,2',4,4',5,6'hexabromodiphenyl ether (3OH-BDE154) $\left(10 \mu \mathrm{g} \mathrm{mL}^{-1}\right.$ in acetonitrile, HBDE-6003S-CN-0.2X), were obtained from AccuStandard, Inc. All other chemicals were of analytical reagent grade. Ultrapure water was used throughout the study for preparing the solutions.

\subsection{Methods}

\subsubsection{Spectral measurements}

The UV-visible absorbance spectra were recorded using a TU1901 absorption spectrophotometer (Beijing Puxi Device Works, Beijing, China) against a solvent as the reference in the wavelength range $300-450 \mathrm{~nm}$.

All the fluorescence spectra were recorded using a RF-5301 PC fluorescence spectrometer (Shimadzu, Japan) equipped with a thermostat bath using $3 / 5 \mathrm{~nm}$ (emission/excitation) slit widths. The fluorescence quenching spectra of HSA were obtained at an excitation wavelength of $280 \mathrm{~nm}$ and an emission wavelength of $300-450 \mathrm{~nm}$. The $\mathrm{pH}$ was measured using a pH-3S digital $\mathrm{pH}$ meter (Shanghai Leici Device Works, Shanghai, China) equipped with a combined glass electrode. All the experiments were carried out at three constant temperatures (Wu et al., 2016; Xiang et al., 2016; Nahid et al., 2013) (291, 298, and $310 \mathrm{~K})$. The ligand solution was rapidly added to the HSA samples at different concentrations (range: $c_{\text {ligand }}=0-10^{-5} \mathrm{M}$; total: 10 samples).

The CD spectra were recorded from 190 to $260 \mathrm{~nm}$ using a Jasco J-820 spectropolarimeter (Tokyo, Japan) equipped with $1.0 \mathrm{~cm}$ quartz cells. For the CD studies, the final concentration of HSA was $10^{-6} \mathrm{M}$, and the concentrations of the ligands were 0 and $2 \times 10^{-7} \mathrm{M}$. The secondary structure was determined using the CDPro software by the CDSSTR method.

\subsubsection{Molecular docking}

The known three-dimensional (3D) crystal structure of HSA (PDB ID: 1AO6, without any crystallized ligand and with a reasonable good resolution of $2.5 \AA$ ) was obtained from the Brookhaven Protein Data Bank (http://www.rcsb.org/pdb/), and its geometry was optimized through the molecular modeling package SYBYL (version x1.1). The water molecules, ions, and original ligands were removed, and hydrogen atoms were added to all the groups with an appropriate geometry within the protein. The initial molecular structures of ligands were constructed based on the structures of similar chemicals obtained from NCBI PubChem Compound (http://www.ncbi.nlm.nih.gov/pccompound/). Then, the ligands were optimized using the Tripos force field and docked into the HSA using the Surflex-Dock program interfaced with SYBYL x1.1. The details and evaluation of docking processing empirical methods have been described in the literature to define a function composed of complexes (Jain, 2007). The top total score conformation of each ligand was selected as the bioactive conformation. Finally, the receptor and ligand were merged to form a complex for the MD simulation and further analysis.

\subsubsection{MD simulation}

Three independent MD simulations ( $20 \mathrm{~ns}$ ) were carried out using the GROMACS 5.0 package in GROMOS96 43a1 force field. The topological parameters of HSA were created. The topological parameters of the ligands were built by the Dundee PRODRG 2.5 server (http://davapc1.bioch.dundee.ac.uk/cgi-bin/prodrg). Then, the complex was immersed in a cubic box (with a Fourier grid of $72 \times 72 \times 72$ ) of extended simple point charge (SPC water) molecules. The solvated system was neutralized by adding sodium ions in the simulation; the entire system was composed of 5843 atoms of HSA. To release the conflicting contacts, energy minimization was first performed using the conjugate gradient method of 1000 steps and then using the steepest descent method for 1000 steps. All the atoms were given an initial velocity, which was obtained from a Maxwellian distribution at the desired initial temperature of $298 \mathrm{~K}$. The density of the system was adjusted during the first equilibration runs under NPT condition by weakly coupling to a bath of constant pressure ( $P=1$ bar) and long range. The electrostatic interactions were evaluated using the particle mesh Ewald 

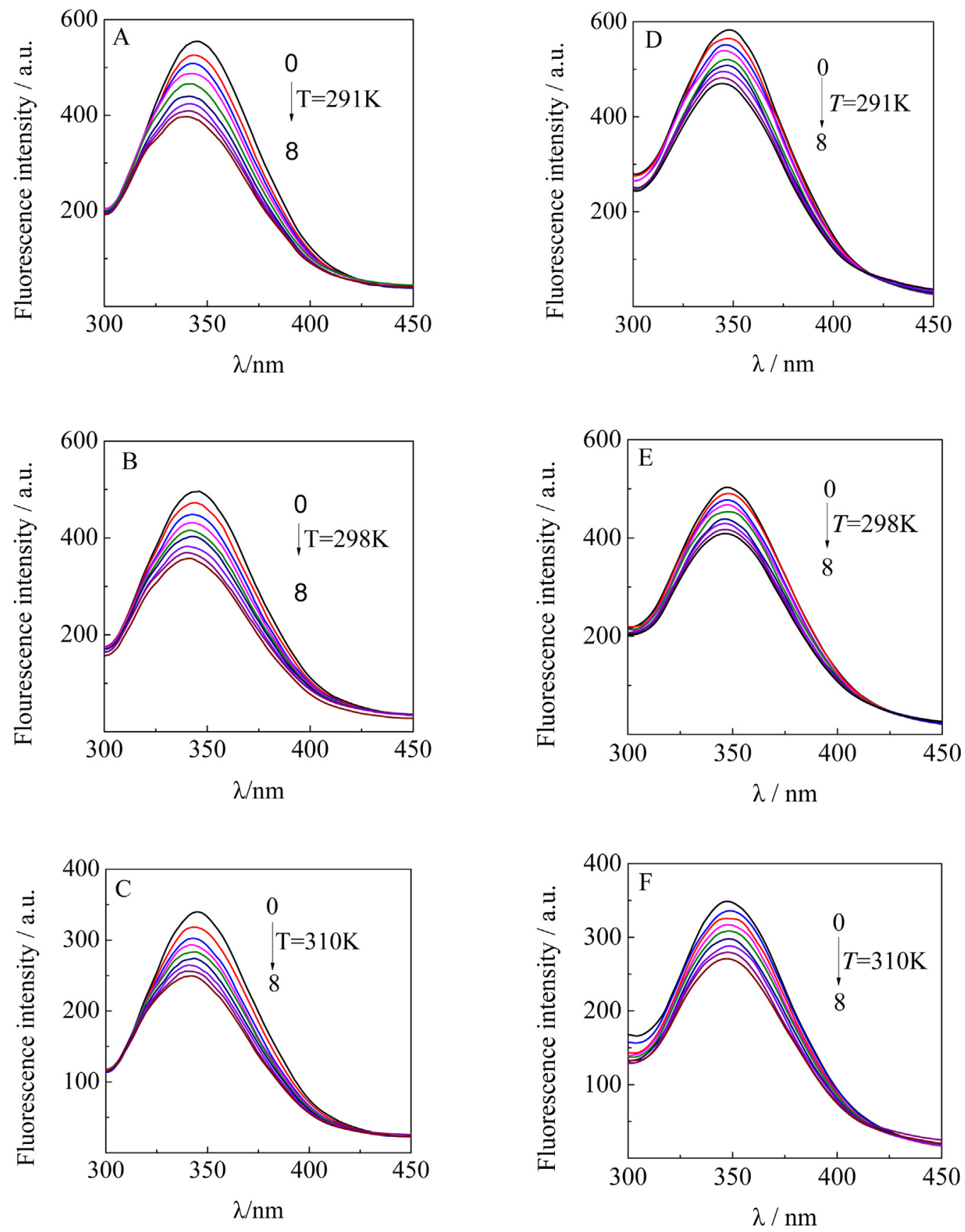

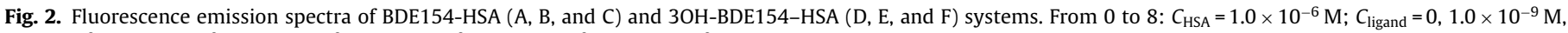
$2.0 \times 10^{-9} \mathrm{M}, 3.0 \times 10^{-9} \mathrm{M}, 4.0 \times 10^{-9} \mathrm{M}, 5.0 \times 10^{-9} \mathrm{M}, 6.0 \times 10^{-9} \mathrm{M}, 7.0 \times 10^{-9} \mathrm{M}$.

(PME). The atom coordinates were recorded every 0.5 ps during the simulation, and the trajectories were analyzed using VMD software.

\section{Results and discussion}

\subsubsection{Fluorescence quenching mechanism}

The typical emission peak of HSA was observed at $362 \mathrm{~nm}$, and the excitation wavelength was $280 \mathrm{~nm}$. This fluorescence of the protein is caused by Trp214 residues only (Vignesh et al., 2016). The maximum fluorescence peak intensity of HSA decreased with the increasing concentrations $\left(10^{-6}-10^{-5} \mathrm{M}\right)$ of ligands with a fixed concentration of HSA $\left(10^{-6} \mathrm{M}\right)$ at $280 \mathrm{~nm}$ (Fig. 2). The change in the intrinsic fluorescence intensity of HSA indicates that the lig- and binding to HSA caused microenvironmental changes in HSA due to the formation of HSA-ligand complexes. Similar fluorescence results were reported for the interactions of different ligand molecules with HSA.

Quenching mechanisms are usually classified as dynamic or static quenching (Huang et al., 2015a,b); they are analyzed using the Stern-Volmer equation, Eq. (1) (Zhang et al., 2014) as follows:

$\frac{F_{0}}{F}=1+K_{\mathrm{q}} \tau_{0}[Q]=1+K_{\mathrm{SV}}[Q]$

$J=\frac{\int_{\infty}^{0} F(\lambda) \varepsilon(\lambda) \lambda^{4} \mathrm{~d} \lambda}{\int_{\infty}{ }^{0} F(\lambda) \mathrm{d} \lambda}$

$R_{0}^{6}=8.79 \times 10^{-25} K^{2} n^{-4} \phi J$ 

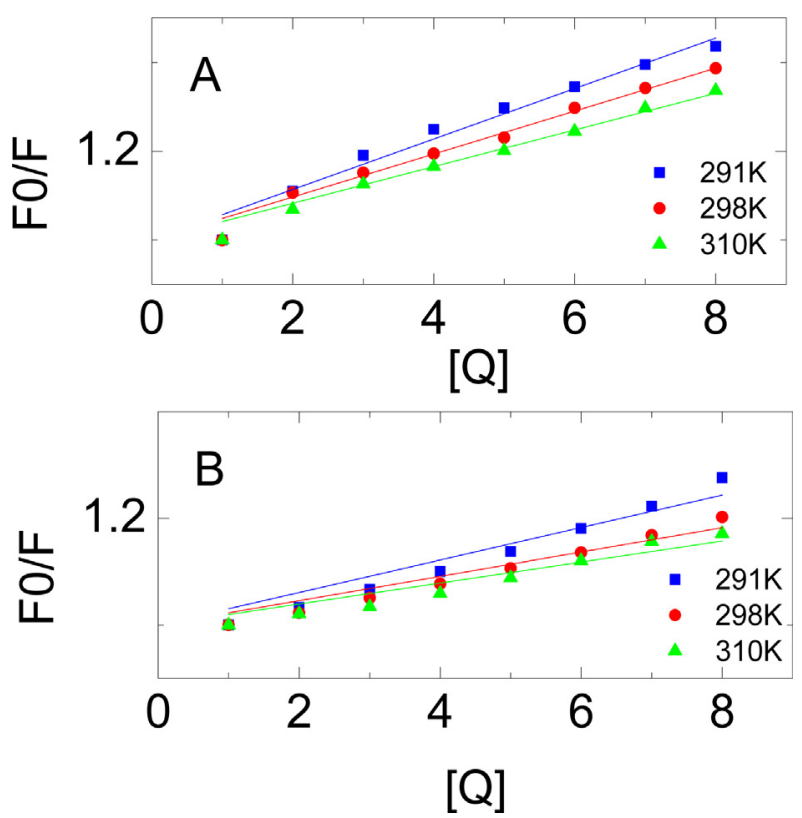

Fig. 3. Stern-Volmer curves of (A) BDE154-HSA and (B) 3OH-BDE154-HSA.

Table 1

Quenching constants of HSA-BDE154 and HSA-3OH-BDE154 at different temperatures.

\begin{tabular}{llll}
\hline Compound & $T(\mathrm{~K})$ & $K_{\mathrm{sv}}\left(\mathrm{L} \mathrm{mol}^{-1}\right)$ & $K_{\mathrm{q}}\left(\mathrm{L} \mathrm{mol}^{-1} \mathrm{~S}^{-1}\right)$ \\
\hline BDE154 & 291 & $5.94 \times 10^{7}$ & $5.94 \times 10^{15}$ \\
& 298 & $5.19 \times 10^{7}$ & $5.19 \times 10^{15}$ \\
$30 H-B D E 154$ & 310 & $4.63 \times 10^{7}$ & $4.63 \times 10^{15}$ \\
& 291 & $3.88 \times 10^{7}$ & $3.88 \times 10^{15}$ \\
& 298 & $2.29 \times 10^{7}$ & $2.29 \times 10^{15}$ \\
& 310 & $2.58 \times 10^{7}$ & $2.58 \times 10^{15}$ \\
\hline
\end{tabular}

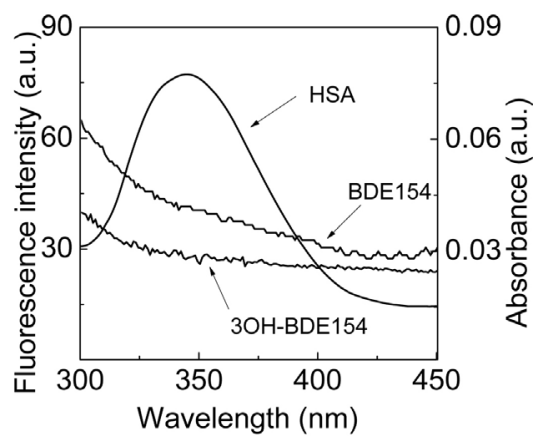

Fig. 4. Overlap of fluorescence spectra of HSA and the absorbance spectrum of BDE154/3OH-BDE154.

$E=1-\frac{F}{F_{0}}=\frac{R_{0}^{6}}{R_{0}^{6}+r^{6}}$

As can be seen from Fig. 3 and Table 1, the values of Stern-Volmer quenching constants $K_{\mathrm{SV}}$ decreased with increasing temperature, and the values of quenching rate constants $K_{\mathrm{q}}$ were much higher than $2.0 \times 10^{10} \mathrm{~L} \mathrm{~mol}^{-1} \mathrm{~s}^{-1}$, indicating that the quenching was static quenching due to the formation of HSA-ligand complexes.

Fig. 4 shows that the fluorescence emission spectra of donors and the absorption spectrum of HSA overlapped. The calculated results of BDE154 and 3OH-BDE154 were $J=1.58 \times 10^{-13}$ and $J=1.26 \times 10^{-13} \mathrm{~cm}^{3} \mathrm{~L} \mathrm{~mol}^{-1}$, critical distance $R_{0}=4.04$ and $3.89 \mathrm{~nm}$, efficiency of transfer $E=0.35$ and 0.11 , respectively. Also, the distance from BDE154 $(r=4.47 \mathrm{~nm})$ and $30 \mathrm{H}-\mathrm{BDE} 154(r=5.48 \mathrm{~nm})$ to

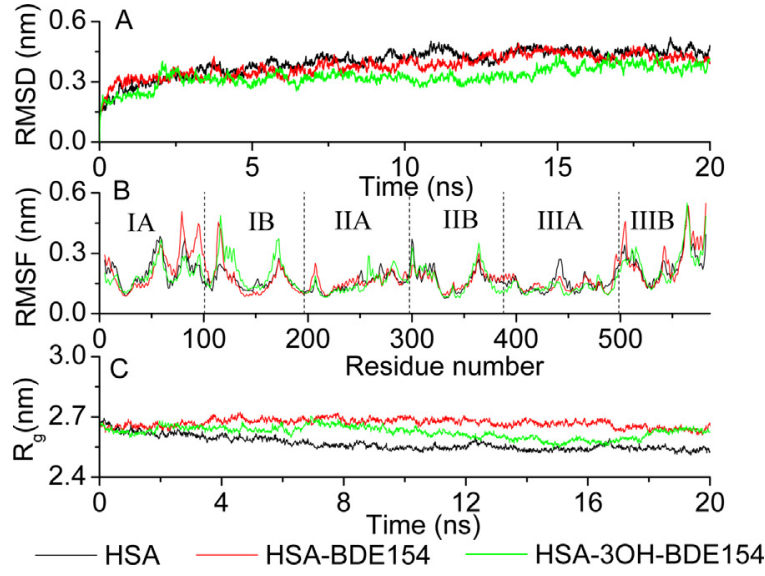

Fig. 5. (A) RMSD values for free HSA (black), HSA-BDE154 (red), and HSA-3OHBDE154 (green) complexes with 20 ns MD simulation. (B) RMSF values for free HSA (black), HSA-BDE154 (red), and HSA-3OH-BDE154 (green) complexes were plotted against the residue numbers. (C) Radius of gyration $(\mathrm{Rg})$ against the simulation time for HSA (black), HSA-BDE154 (red), and HSA-30H-BDE154 (green) complexes.

HSA were less than $7 \mathrm{~nm}$. These results show the effect from the spectral overlap. There is a high probability of energy transfer between the ligands and HSA according to Forster's nonradiative fluorescence resonance energy transfer (FRET) theory (Eqs. (2)-(4)).

\subsection{MD simulation analysis}

MD simulation is an important tool for understanding the physical basis of the structure and function of biological macromolecules. To investigate the stability of the system (HSA, ligand, water, ions, etc.), the properties were examined using root-mean-square deviation (RMSD), root-mean-square fluctuation (RMSF), and radius of gyration $\left(R_{\mathrm{g}}\right)$. The initial structure of the system was first obtained from the molecular docking results and then used in the MD simulations.

The RMSD values of the atoms in the unliganded HSA and HSA-ligand complexes were plotted from 0 to $20 \mathrm{~ns}$ in Fig. 5A. As shown in Fig. 5A, the RMSD values in general stabilized under the MD conditions and only slightly fluctuated around the average values after $10 \mathrm{~ns}$ simulation time. This phenomenon can be explained by the results of the trajectory analysis. The RMSD values of $10-20 \mathrm{~ns}$ were calculated to be $0.45 \pm 0.02 \mathrm{~nm}$ for HSA, $0.42 \pm 0.03 \mathrm{~nm}$ for HSA-BDE154, and $0.35 \pm 0.04 \mathrm{~nm}$ for HSA-3OHBDE154.

In this MD investigation, the obtained averaged RMSF values were based on the 20 ns trajectory data for three different systems and plotted against the residue number (Fig. 5B). As shown in Fig. 5B, the profiles of atomic fluctuation were found to be very similar to each other. This clearly indicates that subdomains IIA and IIIA had the lowest fluctuations, whereas subdomains IA, IB, IIB, and IIIB had the highest fluctuations. In other words, the structure of the main drug-binding site I (subdomain IIA) remained rigid during the simulation. The binding of ligands to HSA mainly affects the loop regions connecting the helices. In this site, the fluctuations are less compared to the drug-binding site II (subdomain IIIA). This study provides evidence that the HSA binding site I specifically interacts with the ligands through the conformational adjustments of the protein structure, in conjunction with the ligand conformational adaptation to the site. Furthermore, it is also consistent with the thermodynamics results obtained from the fluorescence and docking data and the results reported by the MD simulation of different ligand molecules bound to HSA.

Initially, the radius of gyration $\left(R_{\mathrm{g}}\right)$ of unliganded HSA, HSA-BDE154, and HSA-3OH-BDE154 complex was $2.7 \mathrm{~nm}$ (Fig. 5C), 
Table 2

The average fractions of the secondary structure of HSA were estimated by the CDPro software.

\begin{tabular}{|c|c|c|c|c|c|c|}
\hline & \multicolumn{6}{|c|}{ Fraction of secondary structure, \% } \\
\hline & Helix $(r)$ & Helix $(d)$ & Strand $(r)$ & Strand $(d)$ & Turns & Unordered \\
\hline HSA & 23.5 & 17.0 & 15.7 & 0.74 & 15.2 & 20.4 \\
\hline $\mathrm{HSA}+\mathrm{BDE} 154$ & 23.3 & 16.9 & 16.6 & 0.80 & 14.0 & 21.2 \\
\hline $\mathrm{HSA}+3 \mathrm{OH}-\mathrm{BDE} 154$ & 23.8 & 15.7 & 14.0 & 0.73 & 15.5 & 23.6 \\
\hline
\end{tabular}
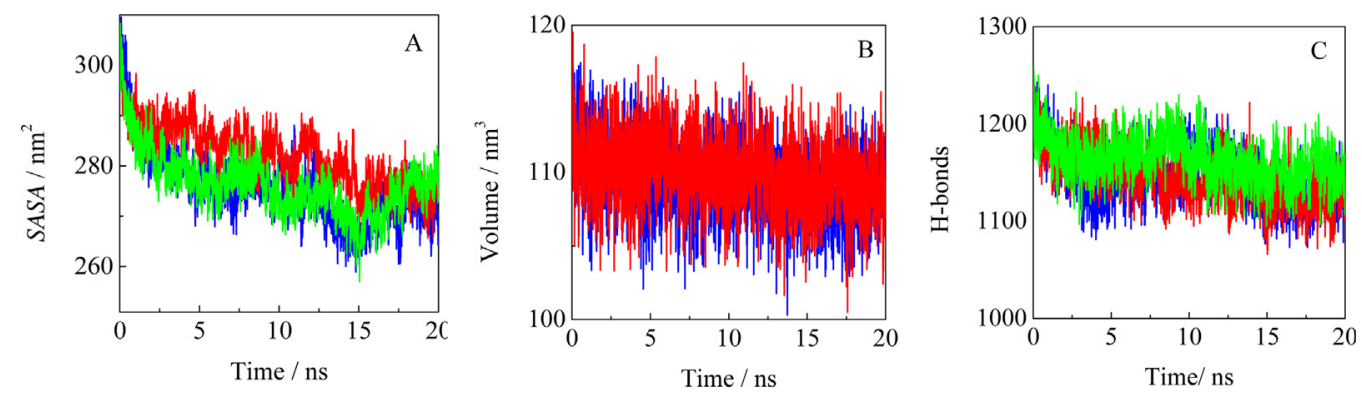

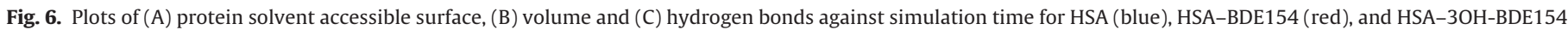
(green).

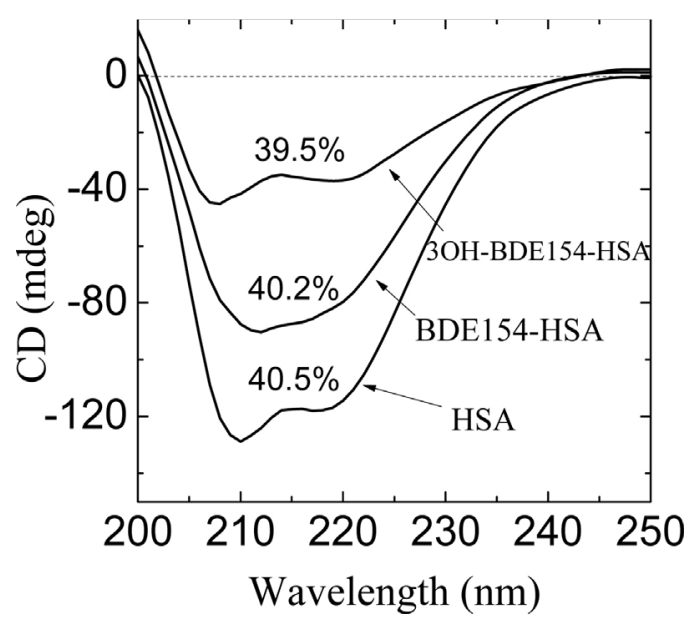

Fig. 7. CD spectra of HSA-BDE154 and HSA-3OH-BDE154 with a protein concentration of $10^{-6} \mathrm{M}$ and ligands concentrations of 0 and $2 \times 10^{-7} \mathrm{M}$.

the same as that reported in the previous report (Sudhamalla et al., 2010). In the three systems, the values of $R_{\mathrm{g}}$ after the $10 \mathrm{~ns}$ MD simulation were $2.55 \pm 0.01,2.66 \pm 0.02$, and $2.60 \pm 0.02 \mathrm{~nm}$, respectively. The results indicate that the systems can achieve equilibrium after $10 \mathrm{~ns}$ in the MD simulation. On the other hand, the $R_{\mathrm{g}}$ values slightly increased upon the binding of BDE154 or 3OHBDE154. The above-mentioned results indicate a change in the microenvironment of HSA when ligands were injected in the MD simulation, strongly supported by the CD spectral data shown in Table 2. Finally, the plots of solvent-accessible surface area (SASA), simulation volume, and hydrogen bond counts (H-bonds) were calculated using gmx hbnum, gmx sasa, and g_energy, respectively (Fig. 6). The data also indicate that as the system stabilized, the SASA, volume, and H-bonds became stable.

\subsection{Secondary structure properties}

To explore the structural changes in more detail, the CD spectra were obtained. Fig. 7 shows the CD spectra of HSA, HSA-BDE154, and HSA-3OH-BDE154 complexes. As shown in Fig. 7, the characteristic features of the $\alpha$-helical structure of protein were observed by negative bands in the UV region at 208 and $222 \mathrm{~nm}$. The addition of BDE154 or 30H-BDE154 significantly increased the ellipticity. This indicates that the structure of HSA was also predominantly $\alpha$-helical because of the similar shape of the CD spectra of unligand HSA and HSA-ligand, and the secondary structure of HSA changed upon binding to BDE154 or $30 \mathrm{H}-\mathrm{BDE} 154$. The CD results were expressed in terms of mean residue ellipticity (MRE) using Eq. (5) (Yang et al., 2016):

$\mathrm{MRE}=\frac{\mathrm{CD}(\text { medg })}{C_{\mathrm{p}} n l \times 10}$

The $\alpha$-helical content of HSA was calculated from the MRE value using the CDPro software in the CDSSTR program. The $\alpha$-helical content differed from that of $40.5 \%$ in free HSA, whereas the contents of helix structure were reduced to 40.2 and $39.5 \%$ when the molar ratio of HSA-ligand complex reached 5:1, indicating the loss of $\alpha$-helical content upon the interaction of the ligands bound with the amino acid residues of the main polypeptide chain of the protein. The data in Table 2 show more details.

The secondary structure of HSA was generated using do_dssp. The results show the $\alpha$-helix content and other secondary structures of the protein in three simulations. As shown in Table 3, the secondary structure patterns of the unligand HSA, HSA-BDE154, and HSA-3OH-BDE154 were compared using the ensemble average divided by the number of amino acids. The analysis shows some changes in the content of secondary structure in the HSA model when ligand molecules were added. Therefore, the principal conformational change proposed for the complexes binding proteins is a simple motion. To consider the dynamic properties of secondary structures, their time evolution was analyzed in detail as shown in Fig. 8.

\subsection{Molecular docking studies}

According to the thermodynamic process, the binding modes were identified for the formation of the complex. Table 4 shows that the thermodynamic parameters, enthalpy $(\Delta H)$, entropy $(\Delta S)$, and Gibbs free energy $(\Delta G)$ of reaction, were calculated according to the Van't Hoff equation. Moreover, the temperature dependence of the binding constant was studied at 291, 298, and $310 \mathrm{~K}$. The HSA thermodynamic parameters of different ligands are listed in Table 4 . A positive $\Delta S$ and a negative $\Delta H$ were commonly observed in the two systems, confirming hydrophobic and specific electrostatic interactions in an aqueous solution. For this purpose, hydrophobic and 
Table 3

Ensemble average of the secondary structure contents in HSA by the do_dssp program.

\begin{tabular}{|c|c|c|c|c|c|c|c|}
\hline & \multicolumn{7}{|c|}{ Fraction of secondary structure, \% } \\
\hline & Coil & B-Bridge & Bend & Turn & A-Helix & 5-Helix & 3-Helix \\
\hline HSA & 14.0 & 0.0 & 9.0 & 6.0 & 70.0 & 0.0 & 1.0 \\
\hline HSA + BDE154 & 13.0 & 0.0 & 8.0 & 7.0 & 71.0 & 1.0 & 0.0 \\
\hline $\mathrm{HSA}+3 \mathrm{OH}-\mathrm{BDE} 154$ & 14.0 & 0.0 & 8.0 & 6.0 & 71.0 & 0.0 & 0.0 \\
\hline
\end{tabular}

A

\section{Secondary structure}

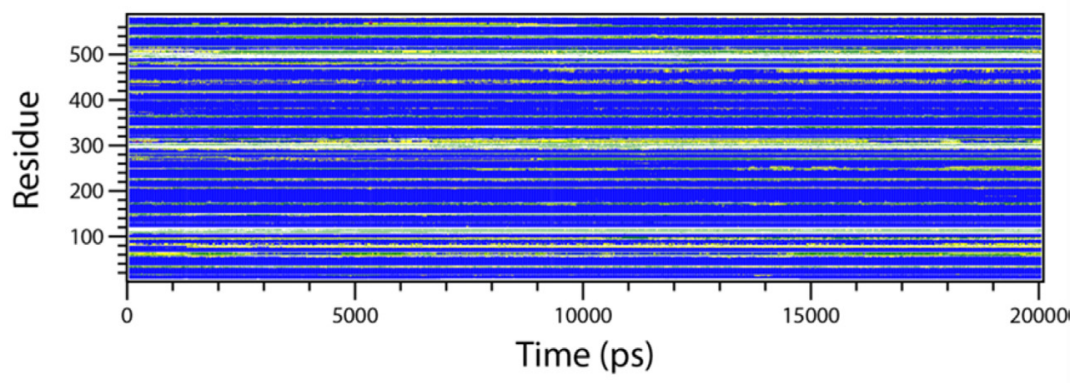

Coil $\square$ B-Bridge $\square$ Bend $\square$ Turn $\square$ A-Helix $\square$ 5-Helix $\square$ 3-Helix

B

\section{Secondary structure}

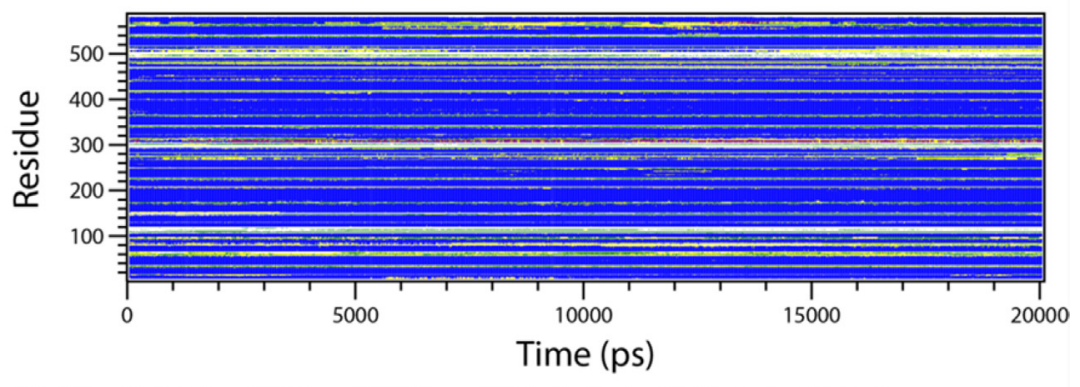

\section{Coil $\square$ B-Bridge $\square$ Bend $\square$ Turn $\square$ A-Helix $\square$ 5-Helix $\square$ 3-Helix}

C

\section{Secondary structure}

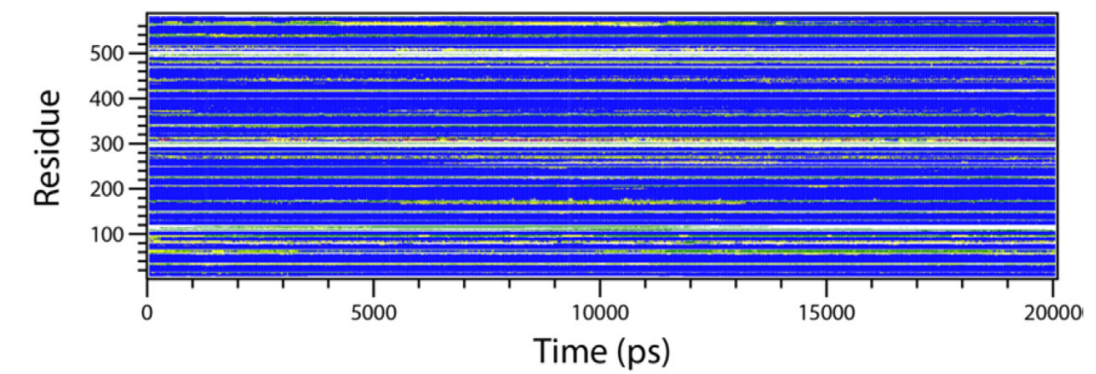

$\square$ Coil $\square$ B-Bridge $\square$ Bend $\square$ Turn $\square$ A-Helix $\square$ 5-Helix $\square$ 3-Helix

Fig. 8. Plots of secondary structure per residue against simulation time (generated using do_dssp) for (A) HSA, (B) HSA-BDE154, and (C) HSA-3OH-BDE154.

Table 4

Thermodynamic parameters at different temperatures.

\begin{tabular}{lllll}
\hline Compound & $T \mathrm{~K}$ & $\Delta H\left(\mathrm{~kJ} \cdot \mathrm{mol}^{-1}\right)$ & $\Delta G\left(\mathrm{~kJ} \cdot \mathrm{mol}^{-1}\right)$ & $\Delta S\left(\mathrm{~J} \cdot \mathrm{mol}^{-1} \cdot \mathrm{K}^{-1}\right)$ \\
\hline BDE154 & 291 & 95.29 & -42.89 & 383.45 \\
& 298 & & -42.38 & \\
& 310 & & -35.89 & \\
$30 H-B D E 154$ & 291 & 61.20 & -41.97 & 336.87 \\
& 298 & & -45.42 & \\
& 310 & & -36.62 & \\
\hline
\end{tabular}

electrostatic interactions probably originated from the binding of protein. Irrespective of BDE154 or 30H-BDE154 in the binding, the binding constants were almost 1.0, indicating only one binding site with HSA.

The molecular docking studies of ligand-HSA interactions were performed using the docking software SYBYL x1.1. According to the $X$-score and competition experiments using ibuprofen and warfarin shown in Table 5, the ligands were commonly bound within the binding pockets of subdomain IIA (site I). The binding affinity of the ligands for site I was predicted by the total docking score. As shown in Table 5 , the docking score for site I increased in the order of BDE154 (2.60) < 3OH-BDE154 (3.14), indicating that the binding affinity of HSA with BDE154 was weaker than 3OH-BDE154. Fig. 9 

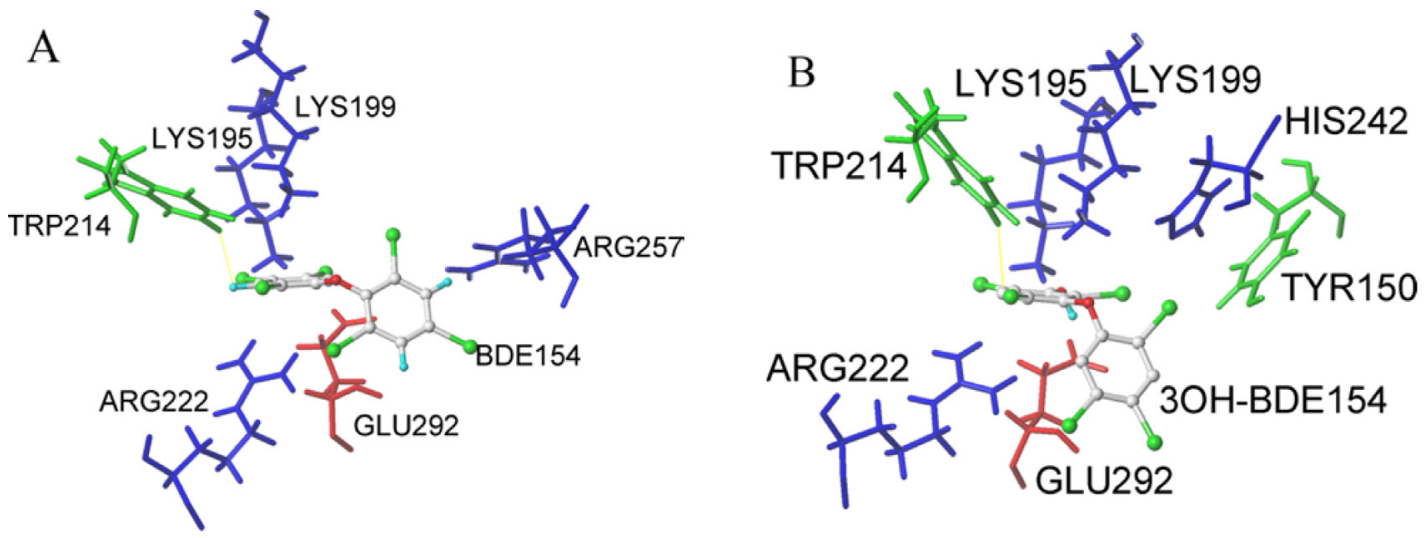

$\mathrm{C}$
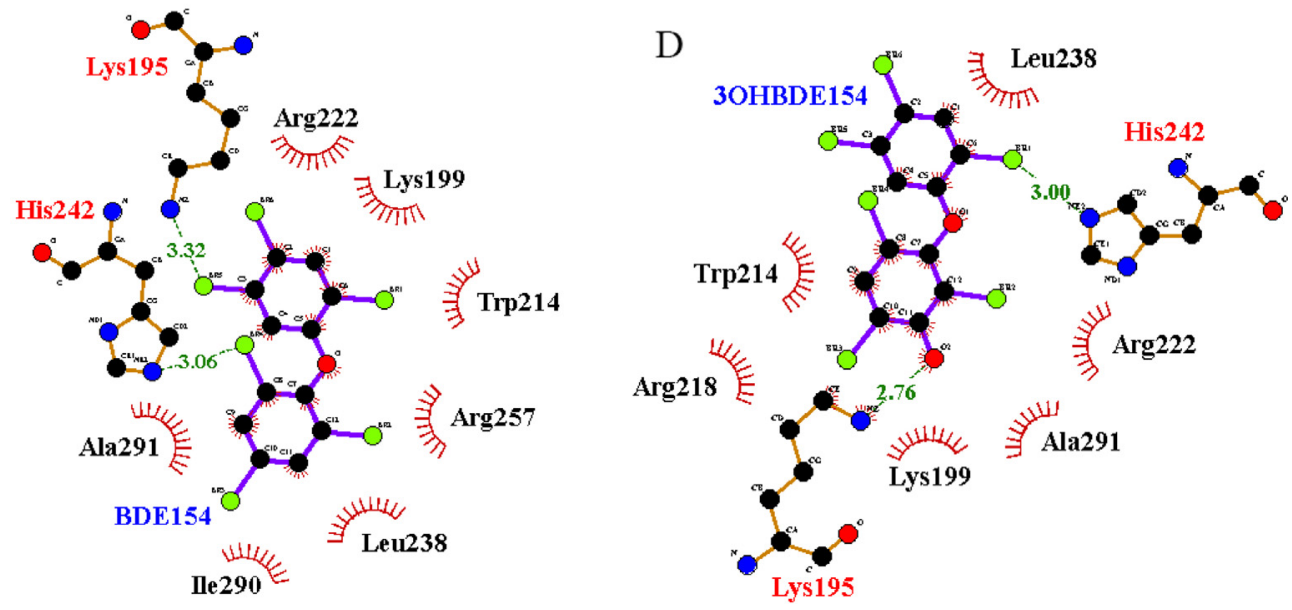

$\mathrm{E}$
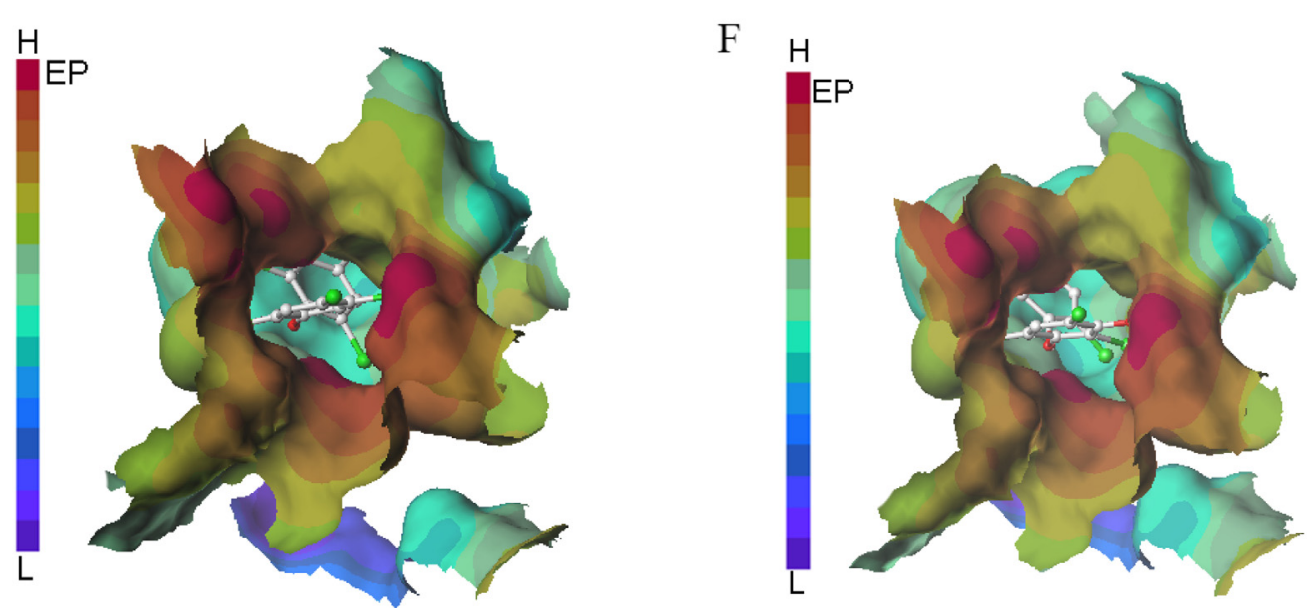

Fig. 9. Molecular docking results of HSA-BDE154 and HSA-30H-BDE154: HSA amino acid residues around 0.5 ̊̊ of (A) BDE154 and (B) 30H-BDE154. Lig plots of (C) HSA-BDE154 and (D) HSA-3OH-BDE154. Electrostatic potentials around (E) BDE154 and (F) 30H-BDE154.

Table 5

Effect of competition experiments and Surflex-docking ( $T=298 \mathrm{~K}$ ).

\begin{tabular}{lllll}
\hline Compound & $K_{\mathrm{a}}\left(\times 10^{6}\right)$ & $\begin{array}{l}\text { Total score } \\
\text { +lbuprofen }\end{array}$ & +Warfarin & \\
\hline BDE154 & 43.7 & 2.27 & 258 & 2.60 \\
3OH-BDE154 & 10.5 & 2.02 & 121 & 3.14 \\
\hline
\end{tabular}

shows the amino acid residues of HSA around $0.3 \mathrm{~nm}$ in the binding of two different ligands.
Fig. 9 clearly shows that the stacking interaction of 3OH-BDE154 and BDE154 with Trp214 had a common feature, except for the $\pi$ stacking with Tyr150. Moreover, as shown in Fig. 9, BDE154 and $30 H-B D E 154$ are both surrounded by the hydrophobic side chains and positively charge residues, such as Trp214, Leu238, Ala291, and Arg222. At the same time, 3OH-BDE154 showed stronger hydrogen bond interactions with a smaller bond length of 0.276 and $0.3 \mathrm{~nm}$. These results indicate that the $30 \mathrm{H}-\mathrm{BDE} 154$ bind site I of HSA had a stronger binding affinity. Further, it was established that HSA-3OH-BDE154 binding involves hydrogen bonding 
and hydrophobic interaction as the main binding forces, and the formation of $\mathrm{H}$-bonds decreased the hydrophilic interaction and increased the hydrophobicity to stabilize the HSA-3OH-BDE154 complexes.

Panels E and F of Fig. 9 show the electrostatic potentials of ligands in the binding pocket of HSA. Several specific electrostatic interactions were also observed, because several ionic charges in the proximity of the ligand play an important role in adjusting the conformation of bound complex. Thus, it can be concluded that the interactions of the two ligands with HSA is mainly hydrophobic, consistent with the thermodynamic results shown in Table 4 obtained from the fluorescence spectra. Moreover, the formation of $\mathrm{H}$-bonds, $\pi$-stacking, and electrostatic interactions play a key role in stabilizing both HSA-BDE154 and HSA-3OH-BDE154 complexes.

\subsection{Discussion}

In the recent years, the research on HSA and ligands is becoming more and more common. The electrochemical method (Huang et al., 2015a,b) and NMR (Maciazek-Jurczyk et al., 2011) method were also applied for the study on HSA and ligands. However, the electrochemical method is difficult to determine some complex oxidation of small molecules (such as PBDEs). NMR measurement requires large molecular weight, and the cost is relatively higher. In addition, the FTIR and Raman spectroscopy (Sharma and Ilanchelian, 2015) were utilized for the analysis of the changes of secondary structure, which is similar to the method combining CD spectroscopy with do_dssp program.

In this study, we studied the BDE154 and 30H-BDE154 binding to HSA using spectroscopy and molecular simulation. There was further insight into secondary structure of HSA-BDE154 and HSA-3OH-BDE154. CD spectroscopy and do_dssp program were used to verify the specified changes in the secondary structure. On the other hand, key residues also were analyzed. BDE154 and 30HBDE154 also formatted H-bond with Lys195 and His242 (Fig. 9C and $\mathrm{D}$ ), the $\mathrm{H}$-bond made stronger interaction with the binding complex.

\section{Conclusion}

The interaction of BDE154 and 3OH-BDE154 with HSA protein was investigated by UV-visible absorbance, fluorescence, $C D$, molecular docking, and MD studies. The results indicate that the single static quenching can be attributed to a series of reactions caused by energy transfer. The competition experiments and molecular docking of BDE154 and 3OH-BDE154 show the main differences in the binding modes of BDE154 or 3OH-BDE154, and the key amino acid residues were located in site I of HSA. From the extended conformation and helix content, the changes in the secondary structures were predicted using the CDPro software and do_dssp program. The simulated cases are consistent with the corresponding experimental data. In particular, the assay of thermodynamic parameters showed electrostatic and hydrophobic interactions as the main binding forces in the complex, and the formation of $\mathrm{H}$-bonds decreased the hydrophilicity and increased the hydrophobicity, thus stabilizing the structures. Finally, all the approaches used in this study indicate that 30H-BDE154 has a stronger binding affinity to HSA than BDE154.

\section{Conflict of interest}

The authors declare that there is no conflict of interest.

\section{Acknowledgments}

This research was financially supported by the National Natural Science Foundation of China (Nos. 21267008) and the Guangxi Natural Science Foundation of China (NO. 2013GXNSF AA019034).

\section{References}

Alecsandra, O.S., Maria, J.T., Alana, M.C.O., Lilian, C.P., Filipe, V.D., Danielle, P.O., Carlos, M.P., Daniel, J.D., 2016. Evaluation of polybrominated diphenyl ether toxicity on HepG2 cells - hexabrominated congener (BDE-154) is less toxic than tetrabrominated congener (BDE-47). Basic. Clin. Pharmacol. Toxicol. 119, 485-497.

Baler, K., Martin, O.A., Carignano, M.A., Ameer, G.A., Vila, J.A., Szleifer, I., 2014. Electrostatic unfolding and interactions of albumin driven by $\mathrm{pH}$ changes: a molecular dynamics study. J. Phys. Chem B 118, 921-930.

Cao, J., Lin, Y., Guo, L.H., Zhang, A.Q., Wei, Y., Yang, Y., 2010. Structure-based investigation on the binding interaction of hydroxylated polybrominated diphenyl ethers with thyroxine transport proteins. Toxicology 277, 20-28.

Carter, D.C., He, X.M., 1990. Structure of human serum-albumin. Science 249, 302-303.

Chen, A., Park, J.S., Linderholm, L., Rhee, A., Petreas, M., DeFranco, E.A., Dietrich, K.N., Ho, S.M., 2013. Hydroxylated polybrominated diphenyl ethers in paired maternal and cord sera. Environ. Sci. Technol. 47, 3902-3908.

Dingemans, M.M.L., Van den Berg, M., Westerink, R.H.S., 2011. Neurotoxicity of brominated flame retardants: (in)direct effects of parent and hydroxylated polybrominated diphenyl ethers on the (developing) nervous system. Environ. Health Perspect. 119, 900-907.

Erratico, C.A., Szeitz, A., Bandiera, S.M., 2013. Biotransformation of 2,2',4,4'-tetrabromodiphenyl ether (BDE-47) by human liver microsomes: identification of cytochrome P450 2B6 as the major enzyme involved. Chem. Res. Toxicol. 26, 721-731.

Ghuman, J., Zunszain, P.A., Petitpas, I., Bhattacharya, A.A., Otagiri, M., Curry, S., 2005. Structural basis of the drug-binding specificity of human serum albumin. J. Mol. Biol. 353, 38-52.

Huang, S., Qiu, H.N., Lu, S.Y., Zhu, F.W., Xiao, Q., 2015a. Study on the molecular interaction of graphene quantum dots with human serum albumin: combined spectroscopic and electrochemical approaches. J. Hazard. Mater. 285, 18-26.

Huang, S., Qiu, H.N., Liu, Y., Huang, C.S., Sheng, J.R., Su, W., Xiao, Q., 2015 b. Molecular interaction investigation between three CdTe: $\mathrm{Zn}^{2+}$ quantum dots and human serum albumin: a comparative study. Colloids Surf. B Biointerfaces 136, 955-962.

Jain, A.N., 2007. Surflex-Dock 2.1: robust performance from ligand energetic modeling, ring flexibility, and knowledge-based search. J. Comput. Aided Mol. Des. 21, 281-306.

La Guardia, M.J., Hale, R.C., Harvey, E. 2006. Detailed polybrominated diphenyl ether (PBDE) congener composition of the widely used penta-, octa-, and deca-PBDE technical flame-retardant mixtures. Environ. Sci. Technol. 40, 6247-6254

Lílian, C.P., Luiz, F.C.M., Alecsandra, O.S., Daniel, J.D., 2014. BDE-154 induces mitochondrial permeability transition and impairs mitochondrial bioenergetics. J. Toxicol. Environ. Health A 77, 24-36.

Maciazek-Jurczyk, M., Sulkowska, A., Rownicka-Zubik, J., Bojkp, B., Szkudlarek-Hasnik, A., 2011. Polypharmacotherapy in rheumatology: ${ }^{1} \mathrm{H}$ NMR analysis of binding of phenylbutazone and methotrexate to serum albumin. J. Mol. Struct. 993, 302-307.

Mao, H.Y., Hajduk, P.J., Craig, R., Bell, R., Borre, T., Fesik, S.W., 2001. Rational design of diflunisal analogues with reduced affinity for human serum albumin. J. Am. Chem. Soc. 123, 10429-10435.

Marchesini, G.R., Meimaridou, A., Haasnoot, W., Meulenberg, E., Albertus, F., Mizuguchi, M., Takeuchi, M., Irth, H., Murk, A.J., 2008. Biosensor discovery of thyroxine transport disrupting chemicals. Toxicol. Appl. Pharmacol. 232, $150-160$.

McDonald, T.A., 2002. A perspective on the potential health risks of PBDEs. Chemosphere 46, 745-755.

Nahid, S., Aref, K., Neda, H.M., 2013. Study on the interaction of the epilepsy drug, zonisamide with human serum albumin (HSA) by spectroscopic and molecular docking techniques. Spectrochim. Acta A Mol. Biomol. Spectrosc. 114, 627-632.

Odusanya, D.O., Okonkwo, J.O., Botha, B., 2009. Polybrominated diphenyl ethers (PBDEs) in leachates from selected landfill sites in South Africa. Waste Manag. 29, 96-102.

Shahlaei, M., Rahimi, B., Ashrafi-Kooshk, M.R., Sadrjavadi, K., Khodarahmi, R., 2015. Probing of possible olanzapine binding site on human serum albumin: combination of spectroscopic methods and molecular dynamics simulation. J. Lumin. 158, 91-98.

Sharma, A.S., Ilanchelian, M., 2015. Comprehensive multispectroscopic analysis on the interaction and corona formation of human serum albumin with gold/silver alloy nanoparticles. J. Phys. Chem. 119, 9461-9476.

Sato, H., Chuang, V.T.G., Yamasaki, K., Yamaotsu, N., Watanabe, H., Nagumo, K., Anraku, M., Kadowaki, D., Ishima, Y., Hirono, S., Otagiri, M., Maruyama, T., 2014 Differential effects of methoxy group on the interaction of curcuminoids with two major ligand binding sites of human serum albumin. PLoS One 9, e87919.

Stapleton, H.M., Eagle, S., Anthopolos, R., Wolkin, A., Miranda, M.L., 2011. Associations between polybrominated diphenyl ether (PBDE) flame retardants, 
phenolic metabolites, and thyroid hormones during pregnancy. Environ. Health Perspect. 119, 1454-1459.

Sudhamalla, B., Gokara, M., Ahalawat, N., Amooru, D.G., Subramanyam, R., 2010. Molecular dynamics simulation and binding studies of beta-sitosterol with human serum albumin and its biological relevance. J. Phys. Chem B 114 9054-9062.

Vignesh, G., Sugumar, K., Arunachalam, S., Vignesh, S., James, R.A., Arunc, R., Premkumarc, K., 2016. Studies on the synthesis, characterization, human serum albumin binding and biological activity of single chain surfactant-cobalt(III) complexes. Luminescence 31, 523-532.

Wan, Y., Choi, K., Kim, S., Ji, K., Chang, H., Wiseman, S., Jones, P.D., Khim, J.S., Park, S., Park, J., Lam, M.H.W., Giesy, J.P., 2010. Hydroxylated polybrominated diphenyl ethers and bisphenol A in pregnant women and their matching fetuses: placental transfer and potential risks. Environ. Sci. Technol. 44, 5233-5239.

Wang, Y., Ni, Y., 2014. New insight into protein-nanomaterial interactions with UV-visible spectroscopy and chemometrics: human serum albumin and silver nanoparticles. Analyst 139, 416-424

Wang Y.F, Liu, S. S, Zhao, HX, Zhao, G.D. Chen, JW, Zhai, G. S., Zhao, H.D. 2016. Polybrominated diphenylethers (PBDEs) and their hydroxylatedmetabolites (OH-PBDEs) in female serum from Dalian. Int. J. Hyg. Environ. Health 219, $816-822$

Wang, Y.P., Zhang, G.W., 2015. Spectroscopic and molecular simulation studies on the interaction of di-(2-ethylhexyl) phthalate and human serum albumin. Luminescence 30, 198-206.

Wu, D., Yan, J., Wang, J., Wang, Q., Li, H., 2015. Characterisation of interaction between food colourant allura red AC and human serum albumin: multispectroscopic analyses and docking simulations. Food Chem. 170, $423-429$.
Wu, Z.W., Yi, Z.S., Dong, L., Zhang, A.Q., 2016. Molecular simulation study of the specific combination between four kinds of phthalic acid esters and human serum albumin. Environ. Toxicol. Pharmacol. 41, 259-265.

Xiang, Y.H., Duan, L.L., Ma, Q., Lv, Z.Z., Zhu, R.H., Zhang, Z.Y., 2016. Fluorescence spectroscopy and molecular simulation on the interaction of caffeic acid with human serum albumin. Luminescence 31, 1496-1502.

Yadav, R., Sengupta, B., Sen, P., 2014. Conformational fluctuation dynamics of domain I of human serum albumin in the course of chemically and thermally induced unfolding using fluorescence correlation spectroscopy. J. Phys. Chem B $118,5428-5438$.

Yang, H.Q., Huang, Y.M., Wu, D., Yan, J., He, J.W., Li, H., 2016. In vitro investigation of the interaction between the hepatitis $\mathrm{C}$ virus drug sofosbuvir and human serum albumin through ${ }^{1} \mathrm{H}$ NMR, molecular docking, and spectroscopic analyses. New J. Chem. 40, 2530-2540.

Zaidi, N., Ahmad, E., Rehan, M., Rabbani, G., Ajmal, M.R., Zaidi, Y., Subbarao, N., Khan, R.H., 2013. Biophysical insight into furosemide binding to human serum albumin: a study to unveil its impaired albumin binding in uremia. J. Phys. Chem B 117, 2595-2604.

Zhang, H., Yu, D.D., Sun, J., Guo, H.Y., Ding, Q.B., Liu, R.H., Ren, F.Z., 2014. Interaction of milk whey protein with common phenolic acids. J. Mol. Struct. 1058, 228-233.

Zota, A.R., Park, J.S., Wang, Y., Petreas, M., Zoeller, R.T., Woodruff, T.J., 2011. Polybrominated diphenyl ethers, hydroxylated polybrominated diphenyl ethers, and measures of thyroid function in second trimester pregnant women in California. Environ. Sci. Technol. 45, 7896-7905.

Zunszain, P.A., Ghuman, J., McDonagh, A.F., Curry, S., 2008. Crystallographic analysis of human serum albumin complexed with 4Z,15E-bilirubin-IX alpha. J. Mol. Biol. 381, 394-406. 